\title{
Ciclo de mejora en el aula virtual aplicado a la asignatura Física General y Biofísica del Grado de Biomedicina Básica y Experimental
}

\section{Improvement Cycle in Virtual Classroom in the subject of General Physics and Biophysics in the Degree of Basic and Experimental Biomedicine}

Lourdes María Varela Pérez

ORCID: https://orcid.org/0000-0002-8875-6042

Universidad de Sevilla

Departamento de Fisiología Médica y Biofísica

Ivarela@us.es

DOI: http://dx.doi.org/10.12795/9788447231003.068

Pp.: 1436-1457 


\section{Breve descripción del contexto}

La asignatura Física General y Biofísica se imparte en el 1o curso del Grado de Biomedicina Básica y Experimental, en la Facultad de Medicina de la Universidad de Sevilla. Es una asignatura troncal de 6 créditos, y se imparte en el 10 cuatrimestre por el departamento de Fisiología Médica y Biofísica.

Debido a la COVID-19, en este curso académico las clases de teoría son virtuales y se imparten a través de la plataforma de enseñanza virtual de la US, y las prácticas son todas presenciales. Las prácticas se han organizado según la disponibilidad de espacios y horarios, en función de todas las asignaturas que corresponden al primer cuatrimestre del primer curso del Grado. Además, se tiene en cuenta que ya se hayan visto los contenidos teóricos relacionados con las mismas.

En este curso hay 54 alumnos matriculados, siendo 7 de ellos repetidores. En la asignatura se imparten conocimientos físicos aplicados a las ciencias de la Salud, para entender las bases físicas del funcionamiento del sistema circulatorio y respiratorio, los principios físicos de radioterapia, así como de algunas de las técnicas de formación de imagen, de terapia y diagnóstico empleadas en Medicina. El interés de la asignatura radica en la adquisición de un mínimo de conceptos físicos que ayuden al estudiante a entender el funcionamiento del organismo, así como las técnicas con las que en un futuro se encontrarán en su carrera profesional. Por lo general, la asistencia a clase es muy alta (unos 50 alumnos), ya que requiere de un alto grado de atención, pues la mayoría de los alumnos no ha cursado Física en el Bachillerato, por lo que se sienten un poco perdidos al inicio del curso. Este Grado registra la segunda nota de corte más alta de la US para el curso 2020/2021, por lo que los alumnos son muy aplicados y la mayoría muy exigentes consigo mismos. 


\section{Diseño completo del ciclo de mejora en el aula}

\section{Mapa de contenidos y problemas centrales}

El Mapa de Contenidos diseñado para desarrollar este CIMA (Figura 1) ha servido para identificar las ideas principales de los temas que lo integran y son esenciales en la asignatura, establecer las relaciones entre los contenidos que lo componen, reorganizarlos para su desarrollo en las sesiones, y establecer las preguntas claves y actividades que servirían de guía a los alumnos. Este mapa fue mostrado a los alumnos al final del CIMA, a modo de ejemplo, para recomendarles que realicen los suyos propios como ayuda al estudio.

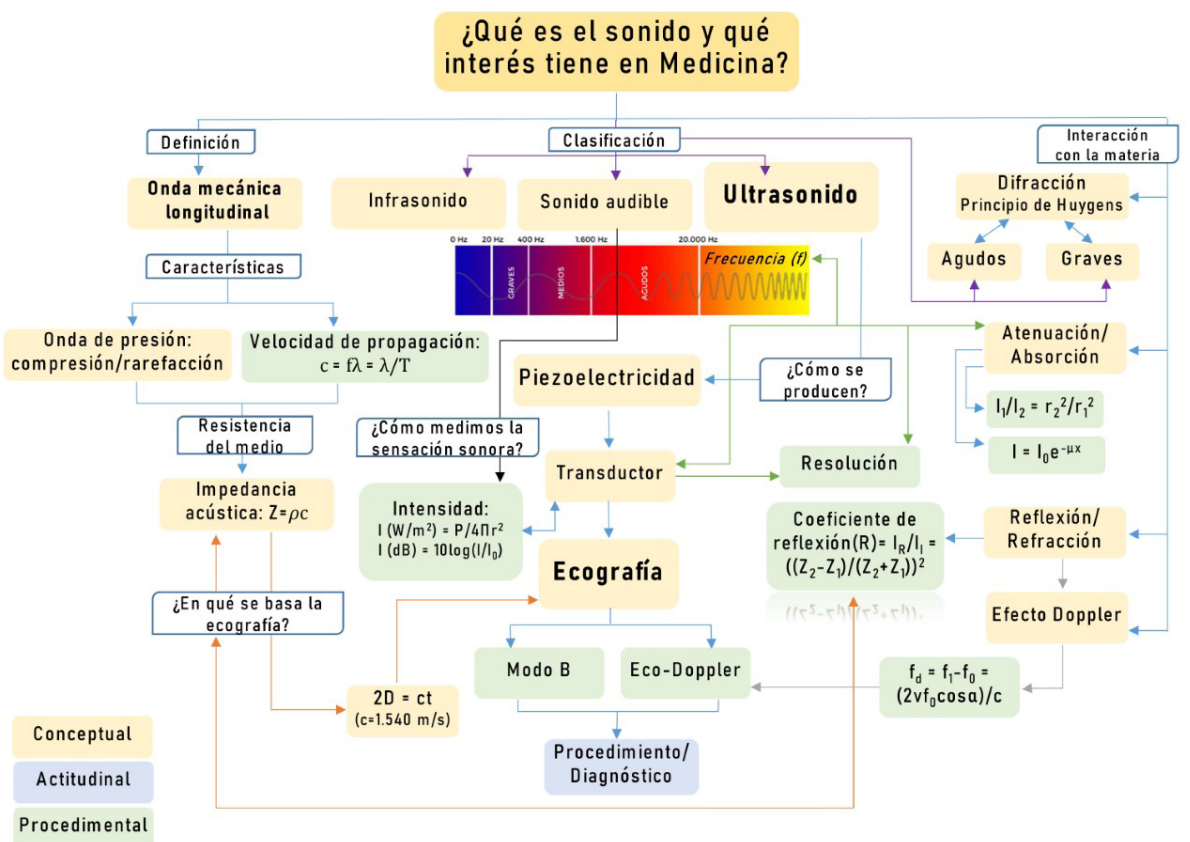

Figura 1. Mapa de contenidos del ciclo de mejora

Ciclos de Mejora en el Aula (2020). Experiencias de Innovación Docente de la US Esta obra se distribuye con la licencia Creative Commons 


\section{Modelo metodológico posible y fichas de actividades secuenciadas}

El principal objetivo de la aplicación de este CIMA consistía en aumentar la participación de los estudiantes en clase para favorecer el pensamiento crítico (Bain, 2005). Dentro de lo que se consideraba posible realizar en la clase, se incorporaron unas preguntas claves que esperaba que el alumno no se hubiera planteado previamente, y a las que se le daría respuesta a lo largo del CIMA. La principal idea se basaba en que los alumnos establecieran sus propias hipótesis para resolver esas preguntas claves y que las compartieran con los compañeros, para servir de punto de partida en el desarrollo de los contenidos (Porlán, 2017). También planteaba que los alumnos fueran quienes realizaran los resúmenes de las sesiones y resolvieran las posibles dudas expuestas por los compañeros, siempre bajo el acompañamiento y supervisión del profesor. Además, se diseñaron actividades grupales para resolver problemas de cálculo relacionados con los contenidos que se estudiaban durante la aplicación del CIMA (Finkel, 2008), y estas se desarrollaban a medida que se avanzaba en el contenido, en lugar de llevarlas a cabo en sesiones separadas y de forma individual, como se había ido realizando anteriormente a la aplicación del presente CIMA. Por último, se incorporaron una serie de actividades recomendadas para realizar entre las sesiones que componen el CIMA, que eran discutidas en clase y servían de apoyo al aprendizaje. La Figura 2 recoge el modelo metodológico del CIMA.

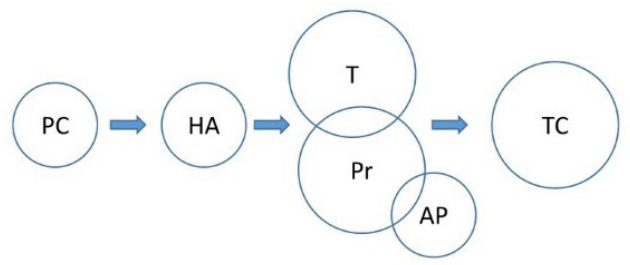

Figura 2. Modelo metodológico implementado en el Ciclo de Mejora. Abreviaturas: AP, actividades propuestas al estudiante; HA, hipótesis de los alumnos; PC: preguntas clave sobre el tema, Pr: problemas de cálculo, T: teoría; TC: taller conceptual.

Ciclos de Mejora en el Aula (2020). Experiencias de Innovación Docente de la US Esta obra se distribuye con la licencia Creative Commons 


\section{Secuencia de actividades}

La Tabla 1 recoge la secuencia de actividades de una de las sesiones impartidas durante el CIMA, a modo de ejemplo de la organización que intenté mantener durante su ejecución, de acuerdo al Modelo Metodológico de la Figura 2. Este CIMA comprendía los tres temas que componen el bloque de la asignatura "Vibraciones y ondas": 1) Movimiento ondulatorio, 2) Ondas sonoras, y 3) Ultrasonidos. Inicialmente, el CIMA estaba diseñado para realizar cuatro sesiones de teoría y ejercicios de cálculos, y una última sesión a modo de taller relacionada con una técnica de diagnóstico y terapia que emplea los ultrasonidos.

Tabla 1. Secuencia de actividades del Ciclo de Mejora para responder a la pregunta:

\section{¿Qué es el Sonido y qué interés tiene en Medicina?}

\begin{tabular}{|c|c|c|c|}
\hline \multicolumn{4}{|c|}{$\begin{array}{c}\text { SESIÓN } \mathbf{2} \\
\text { PC: ¿Por qué el sonido es una onda? ¿Por qué somos capaces de oír unos } \\
\text { sonidos y otros no? ¿Cómo medimos la sensación sonora? }\end{array}$} \\
\hline & $\begin{array}{l}\text { Actividad/Duración/ } \\
\text { Fase del modelo }\end{array}$ & Descripción & Finalidad \\
\hline 1 & $\begin{array}{l}\text { Motivación } \\
15 \text { min } \\
\text { AP }\end{array}$ & $\begin{array}{l}\text { El profesor envía un } \\
\text { correo a los alumnos } \\
\text { con el enlace a una } \\
\text { herramienta que } \\
\text { pueden manipular de } \\
\text { manera virtual con el } \\
\text { fin de identificar las } \\
\text { propiedades de las } \\
\text { ondas }\end{array}$ & $\begin{array}{l}\text { Recordar los } \\
\text { conocimientos } \\
\text { estudiados hasta } \\
\text { el momento } \\
\text { para mejorar el } \\
\text { aprendizaje y ser } \\
\text { hilo conductor de } \\
\text { lo que seguiremos } \\
\text { estudiando }\end{array}$ \\
\hline 2 & $\begin{array}{l}\text { Introducción } \\
5 \text { min } \\
\text { PC }\end{array}$ & $\begin{array}{l}\text { El profesor introduce } \\
\text { la estructura de los } \\
\text { contenidos que se } \\
\text { desarrollarán durante } \\
\text { la sesión (tema } \\
\text { 2.Ondas sonoras), } \\
\text { y propone las } \\
\text { preguntas claves del } \\
\text { tema }\end{array}$ & $\begin{array}{l}\text { Orientar al alumno en } \\
\text { los contenidos y darle } \\
\text { sentido y finalidad a } \\
\text { los mismos }\end{array}$ \\
\hline
\end{tabular}

Ciclos de Mejora en el Aula (2020). Experiencias de Innovación Docente de la US Esta obra se distribuye con la licencia Creative Commons 


\begin{tabular}{|c|c|c|c|}
\hline 3 & $\begin{array}{l}\text { Teoría } \\
15 \text { min } \\
\text { T-HA }\end{array}$ & $\begin{array}{l}\text { El profesor } \\
\text { inicia la sesión } \\
\text { haciendo alusión } \\
\text { a la herramienta e } \\
\text { imparte contenidos } \\
\text { teóricos explicando } \\
\text { conceptos a través } \\
\text { de preguntas a } \\
\text { los alumnos para } \\
\text { resolver las preguntas } \\
\text { claves y saber } \\
\text { si han utilizado } \\
\text { la herramienta. } \\
\text { Ejemplos de } \\
\text { preguntas: } \\
\text { 2a sesión: ¿se puede } \\
\text { propagar el sonido en } \\
\text { el vacío?, ¿por qué no } \\
\text { escuchamos eco en } \\
\text { los espacios vacíos?... }\end{array}$ & 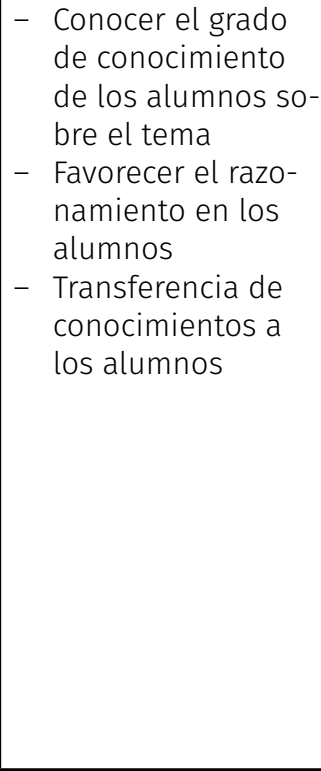 \\
\hline 4 & $\begin{array}{l}\text { Problemas de cálculo } \\
10 \text { min } \\
\text { Pr }\end{array}$ & $\begin{array}{l}\text { El profesor crea } \\
\text { grupos de trabajo } \\
\text { de } 5 \text { alumnos y } \\
\text { les presenta un } \\
\text { problema de cálculo } \\
\text { en cada sesión: } \\
\text { 2a sesión: cálculo de } \\
\text { la longitud de onda } \\
\text { de un sonido en el } \\
\text { aire o sumergido } \\
\text { en el agua con } \\
\text { una velocidad de } \\
\text { propagación diferente } \\
\text { en cada medio }\end{array}$ & $\begin{array}{l}\text { - } \text { Hacer que el } \\
\text { alumno trabaje } \\
\text { el contenido teó- } \\
\text { rico y favorecer su } \\
\text { comprensión } \\
\text { - } \text { Fomentar la co- } \\
\text { municación entre } \\
\text { alumnos } \\
\text { - } \text { Dinamizar la clase } \\
\text { - } \\
\text { El alumno ad- } \\
\text { quiere los conoci- } \\
\text { mientos necesarios } \\
\text { para responder co- } \\
\text { rrectamente a las } \\
\text { preguntas } 3 \text { y } 4 \text { del } \\
\text { cuestionario }\end{array}$ \\
\hline 5 & $\begin{array}{l}\text { Exposición de la } \\
\text { solución } \\
10-15 \text { min } \\
\text { HA-AC }\end{array}$ & $\begin{array}{l}\text { El representante } \\
\text { de un grupo de } \\
\text { trabajo compartirá } \\
\text { la solución con } \\
\text { los compañeros, y } \\
\text { se discutirá con el } \\
\text { profesor y el resto } \\
\text { de equipos, cómo } \\
\text { se ha llegado a esa } \\
\text { solución }\end{array}$ & $\begin{array}{l}\text { - } \text { Compartir el de- } \\
\text { sarrollo del pro- } \\
\text { blema para si } \\
\text { existen diferentes } \\
\text { vías posibles para } \\
\text { resolverlo } \\
\text { - } \text { Favorecer la par- } \\
\text { ticipación del } \\
\text { alumno }\end{array}$ \\
\hline
\end{tabular}

Ciclos de Mejora en el Aula (2020). Experiencias de Innovación Docente de la US Esta obra se distribuye con la licencia Creative Commons 


\begin{tabular}{|c|c|c|c|}
\hline 6 & $\begin{array}{l}\text { Actividad voluntaria } \\
20 \text { min } \\
\text { AP }\end{array}$ & $\begin{array}{l}\text { El profesor propone } \\
\text { otros problemas } \\
\text { de cálculo para } \\
\text { que los realicen de } \\
\text { forma individual } \\
\text { y les propone que } \\
\text { soliciten tutoría para } \\
\text { resolverlos cuando } \\
\text { tengan dudas }\end{array}$ & $\begin{array}{l}\text { - } \text { Seguir trabajando } \\
\text { fuera del aula los } \\
\text { contenidos de- } \\
\text { sarrollados para } \\
\text { favorecer el apren- } \\
\text { dizaje y el razona- } \\
\text { miento individual } \\
\text { - Incitar al uso de } \\
\text { tutorías y cono- } \\
\text { cer mejor a los } \\
\text { alumnos }\end{array}$ \\
\hline 7 & $\begin{array}{l}\text { Conclusiones y dudas } \\
5-10 \text { min } \\
T\end{array}$ & $\begin{array}{l}\text { El profesor implica } \\
\text { a los alumnos en } \\
\text { la identificación de } \\
\text { las principales ideas } \\
\text { aprendidas durante } \\
\text { la sesión y en la } \\
\text { resolución de las } \\
\text { posibles dudas que } \\
\text { sean planteadas }\end{array}$ & $\begin{array}{l}\text { - } \text { Favorecer la capa- } \\
\text { cidad de síntesis } \\
\text { - } \text { Aclaración de } \\
\text { ideas } \\
\text { - Cierre de la sesión }\end{array}$ \\
\hline 8 & $\begin{array}{l}\text { Diario de la sesión } \\
15 \text { min }\end{array}$ & $\begin{array}{l}\text { Anotación de cómo } \\
\text { ha sido el desarrollo } \\
\text { de la sesión }\end{array}$ & $\begin{array}{l}\text { Analizar puntos } \\
\text { fuertes y débiles en } \\
\text { el diseño }\end{array}$ \\
\hline
\end{tabular}

\section{Cuestionario inicial-final}

Antes de comenzar el CIMA que se describe en el presente capítulo, se elaboró un cuestionario de 5 preguntas para conocer los modelos mentales e hipótesis de los alumnos sobre los problemas al inicio. Este mismo cuestionario fue realizado por los alumnos una vez finalizado el CIMA, para conocer la evolución de sus modelos mentales mediante la elaboración de escaleras de aprendizaje (Porlán, 2017).

Las preguntas planteadas en el cuestionario fueron las siguientes:

1. La figura que se muestra a continuación representa los fenómenos que ocurren cuando una onda incide sobre una superficie de separación de dos medios

Ciclos de Mejora en el Aula (2020). Experiencias de Innovación Docente de la US Esta obra se distribuye con la licencia Creative Commons 
diferentes. Rellena los huecos que faltan y explica con tus palabras que significa cada uno de ellos y a qué fenómenos representan.

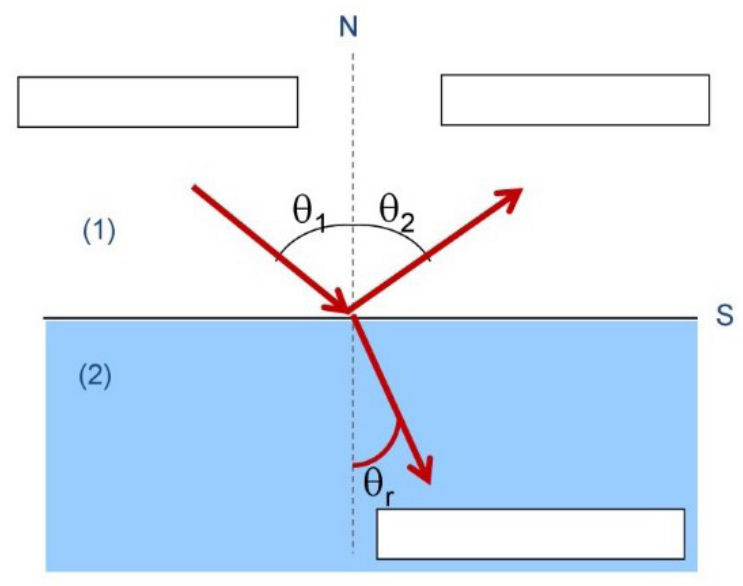

Figura 3. Representación pregunta 1 del cuestionario

2. Con un martillo se golpea un extremo de una barra de cobre de $200 \mathrm{~m}$ de longitud. El sonido se transmite en la barra y llega al otro extremo al cabo de 0,05 s. Calcula la velocidad de propagación del sonido en ese material, explicando el proceso que has seguido y la lógica que tiene.

3. Un barco utiliza un sonar o ecosonda para conocer la distancia que hay hasta el fondo marino para poder navegar. El capitán del Nautilus Star, por prevención, sabe que si la profundidad del fondo marino es menor a $10 \mathrm{~m}$, no debería pasar. Si la velocidad del sonido en el agua salada es aproximadamente de $1.500 \mathrm{~m} / \mathrm{s}$ y la ecosonda capta el sonido reflejado en el fondo cuando han pasado 0,01 segundos desde que se emitió, ¿puede pasar el barco por ahí? Razona tu respuesta.

4. ¿A qué conclusión llegas cuando comparas la velocidad del sonido en el cobre (la que has calculado en la pregunta 2), en el agua salada (enunciado de 
la pregunta 3), y en el aire (c=340 m/s)? Argumenta tu respuesta.

5. Cuando estás parado en un semáforo en rojo y se acerca una ambulancia hacia ti, el sonido de la sirena que te llega cada vez es más agudo. Sin embargo, una vez que pasa de largo y se aleja, ocurre al contrario, el sonido cada vez es más grave. Observa la siguiente representación y explica con tus palabras por qué ocurre eso, y explica algunas aplicaciones que tiene este fenómeno.

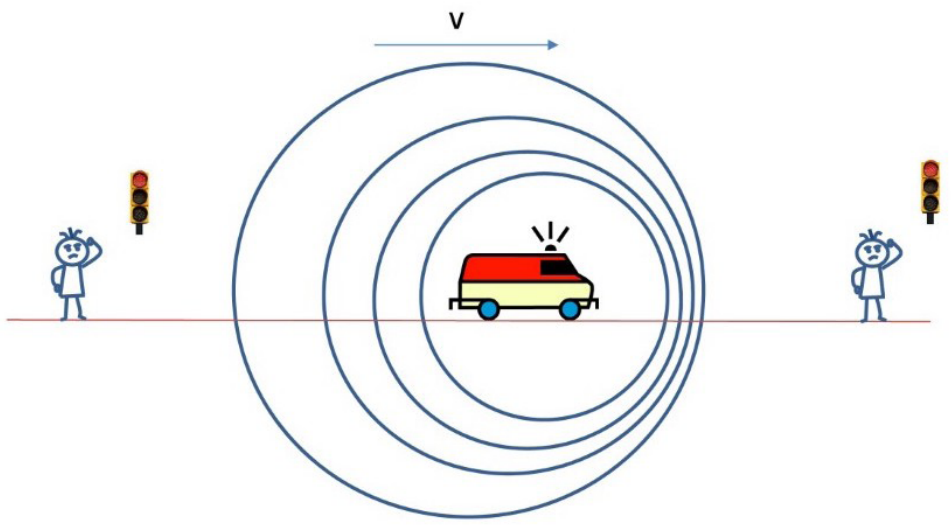

Figura 4. Representación pregunta 5 del cuestionario

\section{Aplicación del Ciclo de Mejora Docente}

\section{Diario de las sesiones}

A continuación, se describe cómo transcurrió el CIMA y las emociones que surgieron durante el mismo. Se describe el relato en primera persona a modo de copia del cuaderno personal que ha sido utilizado para escribir el diario de las sesiones del profesor (Porlán y Martín, 1991):

Antes de comenzar el CIMA en clase, me puse en contacto con los alumnos por correo electrónico para explicarles que en los próximos días íbamos a comenzar un 
CIMA con una duración de 5 sesiones ( 6 horas de duración), para cambiar el modo de impartir las clases que hasta ahora había seguido. En ese mismo correo les facilité el cuestionario inicial para valorar sus conocimientos sobre el bloque que comprendía el CIMA y que posteriormente me permitiría evaluar la evolución de los modelos mentales de los alumnos tras la finalización del CIMA, al realizar por segunda vez el mismo cuestionario durante la última sesión diseñada. De 54 alumnos matriculados en la asignatura, recibí 40 cuestionarios dentro del plazo que había establecido para realizarlo. De esos 40 cuestionarios, he analizado un total de 30 para elaborar las escaleras de aprendizaje.

Todas las sesiones del CIMA se han desarrollado a través de la plataforma Blackborad Collaborate y la asistencia de los alumnos ha sido muy alta, con una media de 50 alumnos.

La sesión inicial del CIMA tuvo una duración de 2 horas, con un pequeño descanso entre horas. Antes de iniciar la sesión, me sentía muy nerviosa debido a las tareas planificadas y a la compartimentación que había organizado para desarrollar los contenidos. Me agobiaba el hecho de no completar el diseño planteado, pues si no lo hacía me iría retrasando en la materia de tal forma que no podría completar el CIMA dentro del esquema inicial estructurado. Ya una vez comenzada la clase, me di cuenta de que estaba relajada, aunque al finalizar la sesión no estaba completamente satisfecha conmigo misma pues había sentido un poco de bloqueo durante la sesión, posiblemente por el estado en el que me había encontrado durante toda la mañana (la sesión tuvo lugar de 3-5 pm).

Para comenzar la sesión, les mostré a los alumnos los temas que íbamos a desarrollar a lo largo del CIMA y que eran los correspondientes al bloque 5 del programa de la 
asignatura. Además, les expliqué el objetivo e interés del estudio del tema que comenzábamos, que abarcaba el movimiento ondulatorio, y les propuse una serie de preguntas a las que daríamos respuesta a lo largo del mismo: ¿qué es una onda y cuáles son sus propiedades?, ¿existen diferentes tipos de ondas?, si en un estadio de fútbol se hace una ola que recorre todas las gradas: ¿eso sería una onda?, ¿qué ocurre cuando la onda cambia de medio?... Aunque forzando un poco la intervención de los alumnos durante el desarrollo de los contenidos teóricos para explicar el movimiento oscilatorio y el ondulatorio, conseguí que participaran durante las explicaciones, de modo que ellos razonaran algunas cuestiones que se planteaban a medida que estudiábamos las características de estos dos movimientos. La primera parte de la sesión comprendió, por tanto, el contenido teórico. Tras el descanso, en la segunda parte de la sesión, organicé a los alumnos en grupos de 4 a 5 (aleatoriamente) para resolver dos problemas de cálculo relacionados con la teoría que acabábamos de ver. Les dejé unos 15-20 minutos para resolverlos, y aunque no conseguí entrar en todos los grupos creados, en los que sí lo hice, puede comprobar que estaban trabajando en los problemas planteados. Transcurrido el tiempo, compartí la pizarra en blanco para resolver los problemas, y logré que dos alumnos representantes se ofrecieran voluntarios para mostrar la solución a la que había llegado su grupo. Esto nos tomó el tiempo restante de la sesión, que finalicé resolviendo algunas dudas relacionadas con el contenido visto.

No logré impartir todo el contenido teórico que había previsto inicialmente, por lo que el contenido de las siguientes sesiones se vio alterado por este retraso. A pesar de ello, me sentí satisfecha de haber logrado la compartimentación de la sesión. Sin embargo, no tuve la misma sensación con mi intervención a lo largo de la sesión, por lo que una vez terminada decidí formar un grupo de trabajo en Outlook con sus correos electrónicos, con el fin 
de realizarles un diario-resumen para resaltar los puntos más importantes que habíamos visto, y que en el fondo tuve impresión de no haber explicado con claridad. Este diario-resumen lo he mantenido durante todo el CIMA. El grupo, además, ha servido como complemento a las tutorías, ya que los correos electrónicos los reciben todos los miembros que componen el grupo, y les sugerí que las dudas que no pudiéramos resolver en las sesiones, las hicieran a través del grupo para que todos los compañeros pudieran tener también esas explicaciones y les sirvieran de ayuda.

Antes del inicio de la segunda sesión, les envié a los alumnos un correo a través del grupo con el enlace a una aplicación en la que podian trabajar diferentes conceptos que habíamos trabajado en la sesión anterior, con objeto de complementar el aprendizaje. Al inicio de la sesión surgieron una serie de preguntas relacionadas con los contenidos de la sesión anterior, y viendo que eso me iba a tomar algo más de tiempo y cambiaría mi esquema inicial, decidí emplear esa oportunidad para fomentar la participación de los alumnos y que ellos fueran los que resolvieran las dudas planteadas por sus compañeros. Al ser las sesiones virtuales, me resultaba muy difícil identificar las sensaciones de los alumnos, y en ese momento les propuse activar las cámaras a los alumnos que iban a participar en la discusión, y además se me ocurrió la idea de que, 4 de ellos (elegidos al azar) activaran sus cámaras en cada sesión, y además de este modo no me sentiría tan sola hablando con la pantalla del ordenador. Pero ya al decir el nombre del alumno en voz alta, recibí excusas para no hacerlo o dejarlo para otra ocasión, ante lo cual realicé un sondeo, y resultó que la mayoría de los alumnos no quería activar su cámara. No puedo definir con una sola palabra cómo me sentí en ese momento (sorprendida, triste, enfadada...) ya que yo tengo siempre la cámara activada y no hay día que los alumnos se olviden de recordarme que active la grabación de la sesión. Aunque con cámaras apagadas, logré

Ciclos de Mejora en el Aula (2020). Experiencias de Innovación Docente de la US Esta obra se distribuye con la licencia Creative Commons 
que dos alumnos me ayudaran en la aclaración de las dudas que habían planteado sobre el contenido teórico y los ejercicios que habíamos realizado. Durante el resto de la sesión, continuamos desarrollando el contenido teórico mediante el debate de las ideas de los alumnos, y finalizamos la sesión identificando las principales ideas que ellos creían necesarias que debían plasmarse sobre el diario-resumen correspondiente. He intentado implicarles en la elaboración del diario-resumen para que los alumnos presten atención durante la clase y al mismo tiempo favorecer su capacidad de síntesis.

Debido a que iba un poco retrasada en cuanto a los contenidos a desarrollar que estaban planteados en el diseño inicial, aprovechando el contacto con los alumnos entre sesiones mediante el diario-resumen, les informé que en las siguientes sesiones debía acelerar el ritmo para lograr completar el CIMA, de modo que, les pedía que las dudas las plantearan a final de la clase, o en el grupo que había creado en la asignatura. También les dije que, si se diera el caso de que el grado de profundidad fuera muy complejo, me pondría en contacto con el alumno en cuestión para realizar una tutoría.

Las siguientes dos sesiones (tercera y cuarta) transcurrieron conforme al diseño planificado en cuanto a compartimentación: preguntas clave-desarrollo de teoría-grupos de trabajo para resolver problemas-discusión y resumen para anotar en el diario-resumen. Aunque para conseguirlo, tuve que dejar muchos de los enlaces a videos y tutoriales que suelo emplear en las explicaciones para que los alumnos los visualizaran en otro momento, ya que, de lo contrario, no podríamos realizar los grupos de trabajo para resolver y discutir los problemas correspondientes a los temas que desarrollamos durante estas dos sesiones (las sesiones 2 y 3 iniciales, se habían transformado en las sesiones 3 y 4). Según un sondeo que había 
realizado previamente, los alumnos consideraban muy necesario realizar problemas en las clases para entender los conceptos, ya que de ese modo tenían mi directa supervisión y guía para resolverlos. En los grupos de trabajo formados en estas sesiones, les pedía en los que entraba que activaran la cámara para poder ponerles caras a sus nombres, a lo cual accedieron y me resultó muy gratificante. Ya que el tiempo de las sesiones no nos permite realizar más de dos problemas por sesión, adjunto a los diarios-resumen les mandaba otros problemas voluntarios relacionados con el contenido, y debido a las tutorías que me han solicitado, he constatado que los han realizado a pesar de no ser una tarea evaluable, comprobando así el interés de los alumnos por la asignatura. Una vez terminado el CIMA, tengo intención de realizar una sesión dedicada únicamente a realizar problemas relacionados con los contenidos que hemos desarrollado, para profundizar sobre ellos y que no quede nada sin resolver.

En la última sesión, comenzamos desarrollando en común la solución para un problema que había planteado como tarea fuera del aula y para el que el $50 \%$ de los alumnos asistentes (47 ese día) reconocía haberlo trabajado. A continuación, terminamos de ver unos últimos conceptos, antes de realizar por segunda vez el cuestionario para valorar la evolución las ideas de los alumnos tras completar el CIMA, como ya ha sido comentado, mediante escaleras de aprendizaje.

Debido a que el desarrollo de los contenidos que había planteado inicialmente para la primera sesión me ocupó también la totalidad de la segunda, el taller que había propuesto inicialmente para cerrar mi CIMA, lo he transformado en un trabajo evaluable, que formará parte de la evaluación continua de la asignatura.

Ciclos de Mejora en el Aula (2020). Experiencias de Innovación Docente de la US Esta obra se distribuye con la licencia Creative Commons 


\section{Evaluación del aprendizaje de los estudiantes}

A continuación, se muestran las escaleras de aprendizaje correspondientes a las preguntas 2 y 5 del cuestionario (Figuras 5 y 6). Del análisis de las escaleras de las 5 preguntas del cuestionario, se deduce que en 4 de ellas se ha logrado una evaluación positiva en las ideas de los alumnos, aumentando los porcentajes de los alumnos que se encontraban en niveles superiores a la finalización del CIMA, y por ello, se han elegido las escaleras de la pregunta 5 como representación de la tendencia general observada tras el CIMA. Sin embargo, el análisis de las escaleras de la pregunta 2 mostró un retroceso en la evolución de las ideas de los alumnos, lo cual se explica con más detalle en el apartado siguiente.

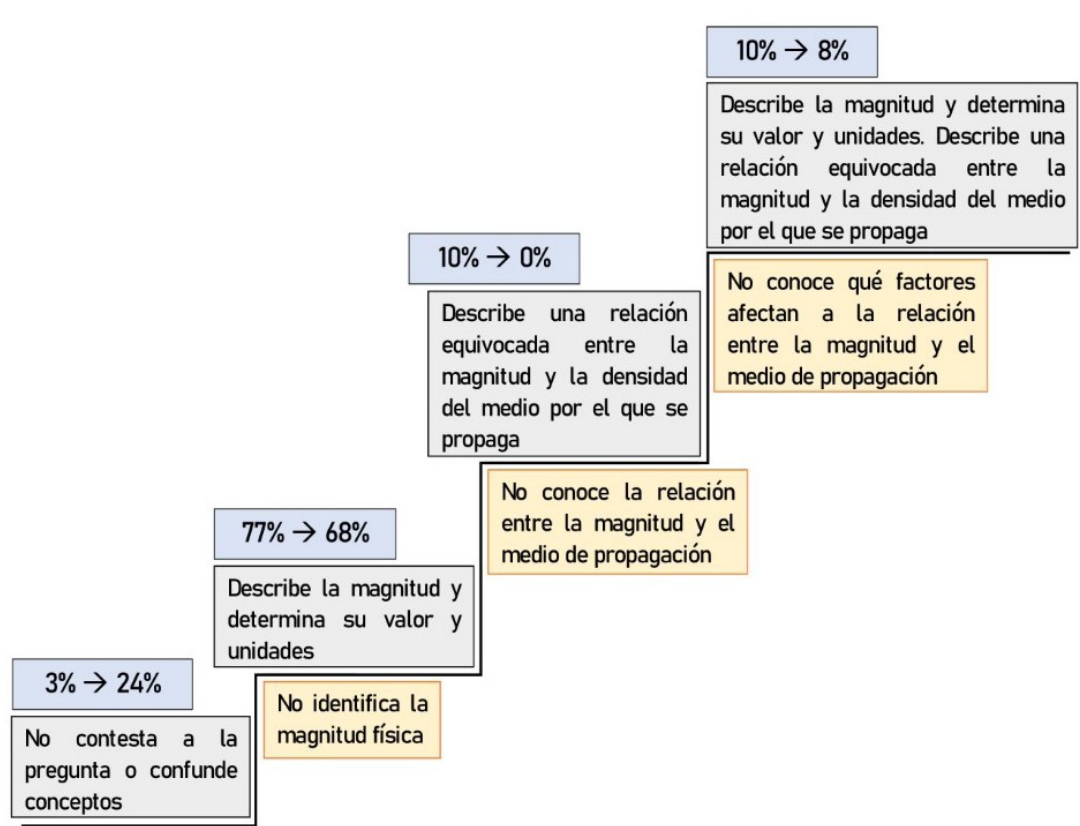

Figura 5. Escaleras de aprendizaje pregunta 2. Comparativa cuestionario inicial y final.

Ciclos de Mejora en el Aula (2020). Experiencias de Innovación Docente de la US Esta obra se distribuye con la licencia Creative Commons 
$70 \% \rightarrow 8 \%$

No contesta a la pregunta - lo hace sin tener relación directa con el fenómeno
$3 \% \rightarrow 76 \%$

Identifica el efecto doppler, describe correctamente el cambio en la longitud de onda que tiene lugar e identifica su aplicación en la ecografía.

No sabe cómo se produce el efecto doppler

Figura 6. Escaleras de aprendizaje pregunta 5. Comparativa cuestionario inicial y final.

\section{Evaluación del Ciclo de Mejora Docente}

La aplicación del CIMA me ha resultado gratificante a la vez que desafiante. Lo que más me ha gustado en todo el proceso, es haber logrado cambiar el modelo que había seguido hasta ahora, basado en una clase magistral, hacia un modelo compartimentado y en el que consigo una mayor participación de los alumnos.

A continuación, voy a ir valorando cada uno de los aspectos que considero más relevantes y necesarios mantener en mi modelo real, y que están relacionados con el diseño y la propia aplicación del CIMA:

- La elaboración del mapa de contenidos y preguntas me ha servido de gran ayuda para identificar los contenidos esenciales en cada uno de los temas que comprendía el CIMA. Sin embargo, para un futuro CIMA debo analizar con detalle que lo que esa sintesis que hago en el mapa se refleje también en la propia presentación

Ciclos de Mejora en el Aula (2020). Experiencias de Innovación Docente de la US Esta obra se distribuye con la licencia Creative Commons 
en la clase y tenga en cuenta posibles imprevistos que puedan ir surgiendo.

- El hecho de haber analizado los cuestionarios (iniciales) antes de comenzar el CIMA, me ha permitido identificar aquellos contenidos a los que debía prestar especial atención para desarrollarlos, ya que, en algunas de las cuestiones planteadas en dicho cuestionario (p.ej. preguntas 2, 3 y 5), había un alto porcentaje de alumnos que no tenía ningún conocimiento relacionado con lo que se cuestionaba en ellas. Sin embargo, tras el análisis de los resultados comparativos en las escaleras de aprendizaje, llego a la conclusión de que he fallado al desarrollar los contenidos relacionados con la pregunta en la que la gran mayoría de los alumnos se encontraba en los escalones superiores inicialmente, al no conseguir una evolución favorable en la misma. Desde mi punto de vista, he debido pensar que no era necesario prestar tanta atención a esos contenidos, y debo tenerlo en cuenta a partir de este mismo momento.

- Aunque hubiera disfrutado más la experiencia siendo las clases presenciales, para interaccionar directamente (presencialmente) con los alumnos, la creación de los grupos de trabajo a la hora de plantear y resolver los problemas, pienso que verdaderamente ha ayudado a los alumnos a tener mayor confianza para exponer sus dudas ante los demás compañeros, ya que he notado una creciente participación en ellos, a medida que transcurrían las sesiones. También esta forma de trabajar en grupos me ha resultado más dinámica que en ocasiones anteriores, en las que los alumnos trabajaron individualmente sobre los problemas, y en las que me resultaba muy difícil encontrar voluntarios para desarrollarlos en la pizarra.

- La realización del diario-resumen tras las sesiones me ha servido para hacer una sintesis de los contenidos de cada una, y que identifico-identifican (los alumnos) 
esenciales, de modo que para un futuro CIMA me puede ayudar a organizar de manera adecuada las sesiones destinadas a los temas trabajados. Además, todos los alumnos han estado muy interesados en recibir los correos y me han comentado que les ayudan mucho a la hora de estudiar los contenidos. De forma paralela, las anotaciones sobre las emociones vividas en mi diario personal me han ayudado a identificar las situaciones en las que me siento más o menos cómoda, y que trataré de trabajar para evitar bloqueos emocionales.

- A excepción de lo comentado junto a la utilidad del cuestionario inicial, en general, las escaleras de aprendizaje me han permitido ver una evolución favorable en las ideas de las que partían los alumnos relacionadas con mayoría de los contenidos. También ligado a esa misma cuestión a la que me refiero que he podido pasar por alto, tengo que mencionar que, aunque haya sido en un pequeño porcentaje, me ha alegrado y motivado aún más a continuar mi desarrollo profesional como docente, observar un desarrollo del razonamiento profundo en unos alumnos, ya que han ido más allá en sus respuestas y sin saberlo, están desarrollando contenido que veremos tras la aplicación del CIMA. Teniendo en cuenta las horas dedicadas a este CIMA en particular, pienso que podría mejorar en un futuro siguiendo mi nuevo modelo didáctico personal.

- Por último, aunque lo haya realizado mediante encuestas y sondeos (herramientas TICS) y no mediante un cuestionario (por cuestiones de tiempo), pretendo incorporar en mi modelo una valoración por parte de los alumnos sobre el diseño y metodología aplicada, para plantear cambios y mejoras en el mismo.

Ciclos de Mejora en el Aula (2020). Experiencias de Innovación Docente de la US Esta obra se distribuye con la licencia Creative Commons 


\section{Cuestiones a mantener y cambios a introducir para un futuro CIMA}

Tras la valoración de la puesta en práctica del CIMA, para uno futuro mantendría todo lo realizado en el CIMA que se ha descrito en el presente capítulo, pero siendo muy consciente de añadir un margen de tiempo para lograr completar en su totalidad el contenido representado en el mapa conceptual. Debo ser muy escrupulosa a la hora de preparar el material de apoyo que empleo en clase para evitar los retrasos que han hecho que tenga que reorganizar el plan inicial.

En el CIMA aquí descrito, la teoría principalmente se ha desarrollado a partir de la formulación de unas preguntas claves a las que los alumnos a las que los alumnos daban respuesta mediante la formulación de hipótesis, que luego eran contrastadas, y las actividades que se han realizado de manera grupal han sido aquellas que se referían al desarrollo de problemas y cálculo. De forma que para mi futuro CIMA aspiro trabajar de manera grupal tanto la teoría como los problemas, todo ello siempre bajo mi guía y supervisión, y teniendo muy presente el capítulo ¿Cómo dirigen la clase?, del libro Lo que hacen los mejores profesores universitarios (Bain, 2005).

Por último, y como ya he comentado anteriormente, introduciría la elaboración de un cuestionario de evaluación del CIMA y del profesor, para que los alumnos lo respondieran una vez finalizado el CIMA, con el fin de conocer aquellos aspectos que se podrían mantener, cambiar o incluir, con el fin de promover el aprendizaje crítico.

Ciclos de Mejora en el Aula (2020). Experiencias de Innovación Docente de la US Esta obra se distribuye con la licencia Creative Commons 


\section{Aspectos de la experiencia que se pretenden incorporar a toda la práctica docente habitual}

Puesto que los resultados han sido en general buenos, los aspectos que se deben mantener son: evaluación inicial de sus modelos mentales con tiempo suficiente para analizar y poder incorporar los cambios necesarios en la docencia, compartimentación de la clase, planteamiento de problemas de forma grupal a medida que se desarrollan los contenidos, mejorar el diseño de mapas de contenidos y la integración de la teoría y la práctica para abordarlo, hacer trabajar al alumno dentro y fuera de clase y usar diferentes herramientas para mantener su motivación.

\section{Principios Didácticos}

Para finalizar, describo brevemente los principios didácticos que han guiado la experiencia presente y que deben permanecer en el futuro, basados en la experiencia y aprendizaje alcanzados en este CIMA y en las lecturas de los trabajos ya citados, además de los de de-Alba-Fernández y Porlán (2020), y Gibbs (SEDA, 2014):

- Conexión con las ideas previas. Ya en la elaboración del cuestionario inicial intenté que con sus respuestas buscaran entre sus conocimientos aquellas ideas de las que ya disponían para desarrollar otras nuevas, lo que ayudaría a ampliar sus modelos mentales iniciales.

- Tomar en consideración los modelos mentales iniciales de los alumnos para adaptar los contenidos y la manera de abordarlos. El análisis de los cuestionarios iniciales me permitió identificar patrones entre los modelos de los alumnos, y a su vez, reconocer modelos mentales que eran la suma de otros. Esto me permitió, al mismo tiempo, definir posibles obstáculos sobre los que tenía que actuar para proporcionar a los alumnos el mismo nivel de aprendizaje. 
- Fomentar el pensamiento crítico natural y hacer reflexionar a los alumnos. Entre las actividades que se han realizado en el CIMA con este fin, se incluyen: formulación de preguntas clave que han servido de guía en las explicaciones, participación en debates para reflexionar sobre los contenidos, y análisis de los videos y el material proporcionado a los alumnos para trabajarlo fuera del aula.

- Sistematización de la teoría y la práctica, y también de los contenidos para que estuvieran ordenados de forma que lo posterior se basara en lo anterior, favoreciendo así, la reflexión en los alumnos y la asociación de la información que reciben, y con ello, la evolución de sus modelos mentales.

- Incorporación de actividades para estimular la motivación en los alumnos: actividades propuestas para realizar fuera del aula (problemas, enlaces a videos y tutoriales), diario-resumen de las sesiones, y creación de grupo de trabajo en Outlook para complementar las tutorías.

- Incluir espacios para aprender a ser-conocer, entendiendo la Universidad como espacio de formación integral de las personas. Durante el CIMA se favoreció la interacción entre alumnos en grupos pequeños para dar respuesta a los problemas. Al ser un curso virtual, a los alumnos les ha faltado ese vínculo de compañeros, y al menos así, considero que han podido socializar entre ellos, ya que disponían de suficiente tiempo para desarrollar los problemas planteados. 
Palabras clave: Física General y Biofísica, Biomedicina Básica y Experimental, docencia universitaria, experimentación docente universitaria

Keywords: General Physics and Biophysics, Basic and Experimental Biomedicine, university teaching, experimental university teaching

\section{Referencias bibliográficas}

Bain, K. (2005). Lo que hacen los mejores profesores universitarios. Valencia: Publicaciones de la Universidad de Valencia.

De-Alba-Fernández, N. y Porlán, R. (2020). Docentes Universitarios. Una formación centrada en la práctica. Madrid: Morata.

Finkel, D. (2008). Dar clase con la boca cerrada. Valencia: Publicaciones de la Universidad de Valencia.

Gibbs, G. (2014). 53 Powerful Ideas All Teachers Should Know About. SEDA. Recuperado de https://www.seda. ac.uk/53-powerful-ideas

Porlán, R. y Martín, J. (1991). El diario del Profesor. Un recurso para la investigación en el aula. Sevilla: Díada.

Porlán, R. Coord. (2017). Enseñanza universitaria. Cómo mejorarla. Madrid: Morata.

Ciclos de Mejora en el Aula (2020). Experiencias de Innovación Docente de la US Esta obra se distribuye con la licencia Creative Commons 